OPTO-ELECTRONICS

INVITED PAPER

\title{
All-optical switching and passive mode-locking based on non-linear polarization rotation in a semiconductor quantum well amplifier
}

\author{
C. C. YANG, CHOONG-WEN LAY, MING-SHAN LIN, \\ DING-WEI HUANG \\ Institute of Electro-Optical Engineering and Department of Electrical \\ Engineering, National Taiwan University, Taipei, Taiwan, ROC
}

Received 13 December 1995; accepted 25 March 1996

Owing to differences in the selection rules of electron transitions between TE and TM modes in a quantum well structure, an optical amplifier with such a structure manifests linear birefringence and anisotropy of gain-absorption saturation. The anisotropy leads to the evolution of power-dependent polarization in the optical amplifier. We demonstrate experimentally efficient non-linear self-switching based on this phenomenon in an InGaAs/GaAs single quantum well amplifier. Also, by utilizing the non-linear polarization evolution, we show numerically passive mode-locking of a semiconductor laser with a ring cavity.

\section{Introduction}

Non-linear optical phenomena can find applications in many areas, including all-optical switching and passive laser mode-locking. For either applications, optical non-linearities can be classified into two categories: fast non-linearities, such as Kerr-like effects in optical fibres and semiconductors below bandgaps, that have decay times of the order of $100 \mathrm{fs}$; and slow non-linearities, such as gain-absorption saturation in semiconductors, that have decay times of the order of $1 \mathrm{~ns}$. Generally speaking, slow non-linearities are much stronger than fast non-linearities.

For all-optical switching, the phenomena of non-linear Fabry-Pérot resonance and nonlinear directional coupling have been widely used. All optical switching devices based on non-linear Fabry-Pérot resonance were usually made of semiconductors near bandgaps with pump-probe configurations [1]. Those based on non-linear directional coupling were made of semiconductors near bandgaps [2], semiconductors near half bandgaps [3] and optical fibres [4]. Efficient self-switching has been widely observed. Besides, non-linear polarization rotation in a birefringent waveguide has recently attracted attention for all-optical switching $[5,6]$. 
Because of the non-linear anisotropy, evolution of polarization in such a waveguide (passive or active) depends on the input power. Both optical fibres and semiconductors near half bandgaps have been considered for this purpose.

For laser passive mode-locking, fast non-linearities have led to Kerr-lens mode-locking for Ti : sapphire and other solid-state lasers, additive-pulse mode-locking for colour-centre and other lasers, and polarization additive-pulse mode-locking for Er-doped fibre lasers. Slow non-linearities, such as saturable absorption in dyes and semiconductors, have been widely used for passively mode-locking various lasers, including dye lasers, colour-centre lasers, semiconductor lasers, etc. Colliding-pulse mode-locking is a mechanism that has been developed, based on saturable absorption.

Although all-optical switching based on non-linear polarization rotation has been implemented in optical fibres and semiconductors near half bandgaps with fast nonlinearities $[5,6]$, a similar device based on slow non-linearities has not been developed yet. Meanwhile, although passive mode-locking for fibre lasers based on non-linear polarization rotation (polarization additive-pulse mode-locking) has been implemented [7,8], passive mode-locking based on slow non-linearity induced polarization rotation has never been reported. In this paper, we present the experimental results of efficient non-linear polarization switching in a single quantum well optical amplifier and the numerical results of passive mode-locking of a semiconductor quantum well laser based on the observed phenomena. In a quantum well amplifier, evolution of power-dependent polarization is attributed to the anisotropy of gain-absorption saturation between the TE and TM polarization modes [9]. This anisotropy comes from differences in the selection rules of electron transitions between the two polarization modes.

This paper is organized as follows: In Section 2, the experimental procedure and results are described. Then, we discuss the theoretical model and the numerical results used to explain the experimental data in Section 3. Simulations for a passively mode-locked semiconductor laser are presented in Section 4. Finally, conclusions are drawn in Section 5.

\section{Experimental procedure}

The sample used in our experiments was an InGaAs/GaAs single quantum well amplifier; its structure is shown in Fig. 1. The amplifier consisted of a $10 \mathrm{~nm} \mathrm{In}_{0.2} \mathrm{Ga}_{08}$ As well sandwiched by two $40 \mathrm{~nm}$ GaAs barriers, $\sim 200 \mathrm{~nm}$ graded-index separate confinement layers with $\mathrm{Al}_{x} \mathrm{Ga}_{1-x} \mathrm{As}(x=0.4-0)$ and $\mathrm{Al}_{04} \mathrm{Ga}_{0,6}$ As cladding layers. Electroluminescence of the sample peaked at 974 and $940 \mathrm{~nm}$ in the TE and TM modes, respectively. The electroluminescence indicates the energy differences between the heavy-hole and light-hole sub-bands. The peak of the TM electroluminescence was one order of magnitude weaker than that of the TE mode, showing that far fewer holes existed in the light-hole band for interband transitions compared with the heavy-hole band. The sample lased at a threshold current of $120 \mathrm{~mA}$ at $974 \mathrm{~nm}$. The optical signals in the amplifier were laterally confined to a broad area with a $50 \mu \mathrm{m}$ ridge.

The experimental set-up is shown in Fig. 2. We used a picosecond Ti : sapphire laser as our light source, which was pumped by an Argon laser. The optical signals passed through a halfwave plate and through a Glan-Laser polarizer; these were used to control input polarization and the power level, before they were end-coupled into the sample with a $\times 40$ objective lens. At the output end, the signals were recollimated with another $\times 40$ objective lens. The output signals from the sample were separated by a polarization beam splitter with a $30 \mathrm{~dB}$ extinction ratio and were collected by two silicon detectors.

Figure 3 compares the output polarization ellipse of a continuous wave (CW) signal with that of a picosecond input signal of the same average power. To obtain the results, a $\mathrm{CW}$ signal and 


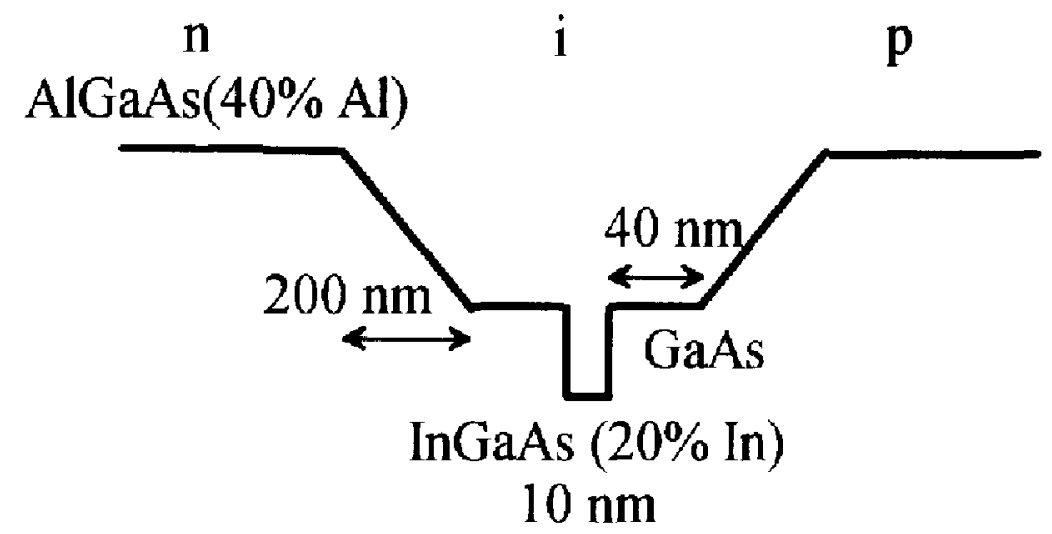

Figure 1 Structure of the inGaAs-GaAs single quantum well amplifier.

a pulse of $3 \mathrm{ps}$ width at $940 \mathrm{~nm}$, both with an average power of $70 \mathrm{~mW}$ and an input polarization of $15^{\circ}$ from the TE direction, were used. The injection current was zero, implying that the optical non-linearity involved was absorption saturation in both polarization modes. Although the two kinds of signals had the same average power, the peak power of the picosecond signal was far greater than that of the $\mathrm{CW}$ signal. In this situation, picosecond and $\mathrm{CW}$ signals experienced different amounts of absorption saturation and ended up with different output polarization ellipses.

In Fig. 4, the output power versus the input power are plotted for the situation when a picosecond signal at $940 \mathrm{~nm}$, with linear input polarization $15^{\circ}$ from the TE direction, was coupled into the amplifier with a $20 \mathrm{~mA}$ injection current. The effective input power value was obtained by estimating the effective input coupling efficiency to be $1.2 \%$. In this estimation, the throughput of the low input part in the TM mode was used to calibrate the effective input coupling efficiency, and hence the linear gain in the TM mode was included in the effective input power. As shown in Fig. 4, the TE curve concaves upwards indicating absorption saturation and, in contrast, the TM curve concaves downwards indicating gain saturation. When the input power level is low, the major part of the output power appears in the TM polarization

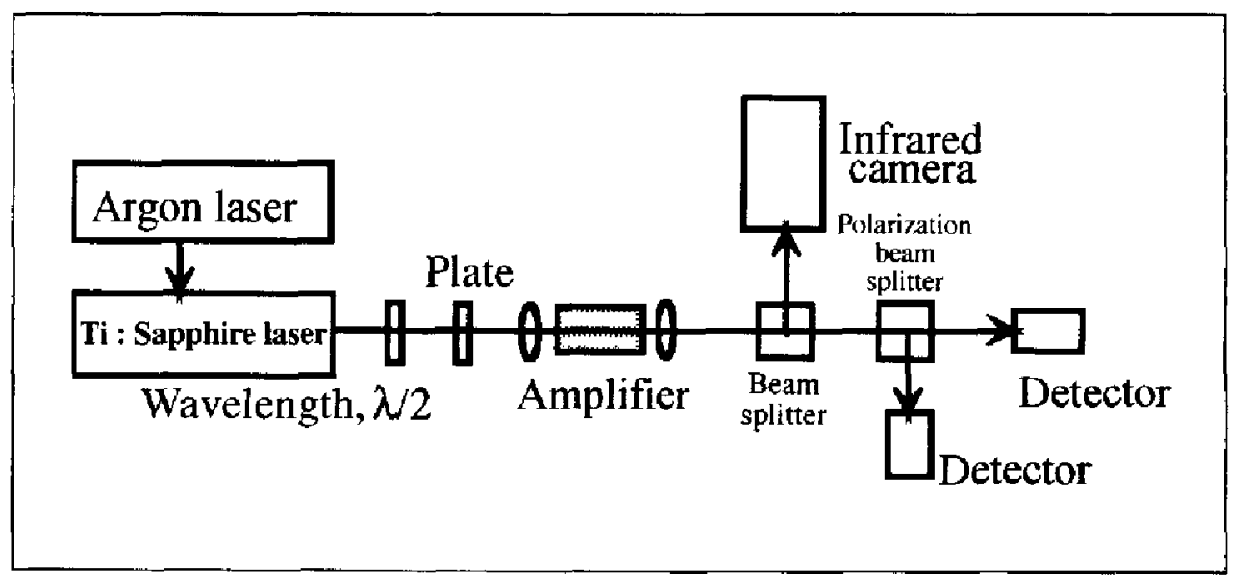

Figure 2 Experimental set-up for the observation of power-dependent polarization rotation. 


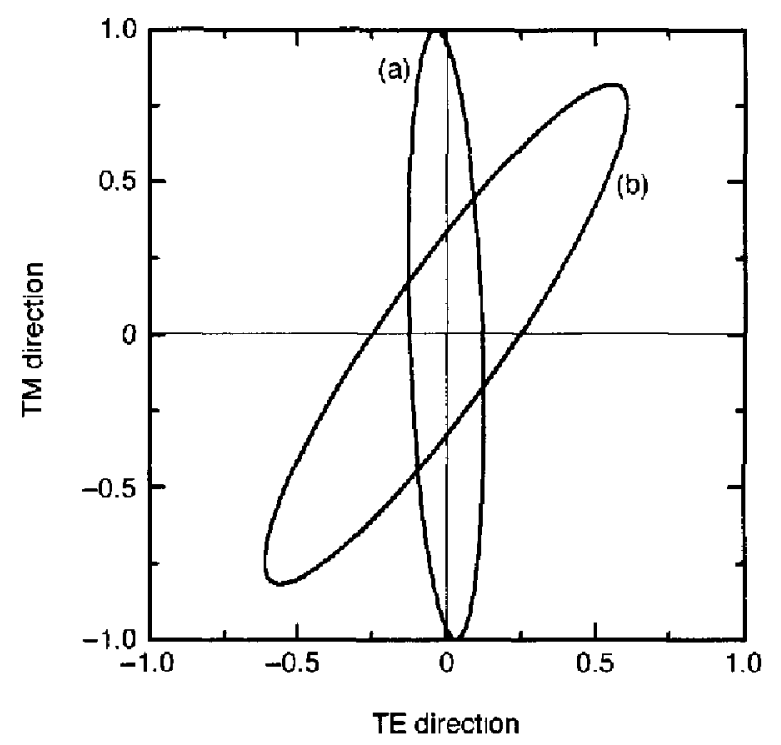

Figure 3 Comparison between the output polarization ellipses of (a) the $\mathrm{CW}$ and (b) the picosecond signals at $940 \mathrm{~nm}$ with zero injection current and $70 \mathrm{~mW}$ average power. The linear input polarization angle is $15^{\circ}$ from the TE direction.

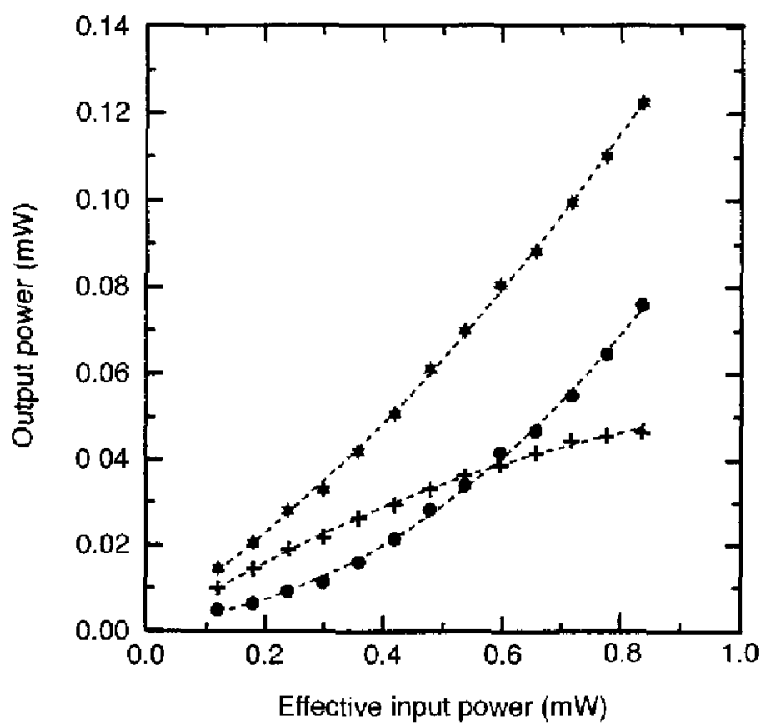

Figure 4 Total output power and the components in the (O) TE and (+) TM modes versus the effective input power level for the picosecond signal at $940 \mathrm{~nm}$ with $20 \mathrm{~mA}$ injection current. The linear input polarization angle is $15^{\text {s }}$ from the TE direction. (- - -) second-order polynomial fit. (1) total. 


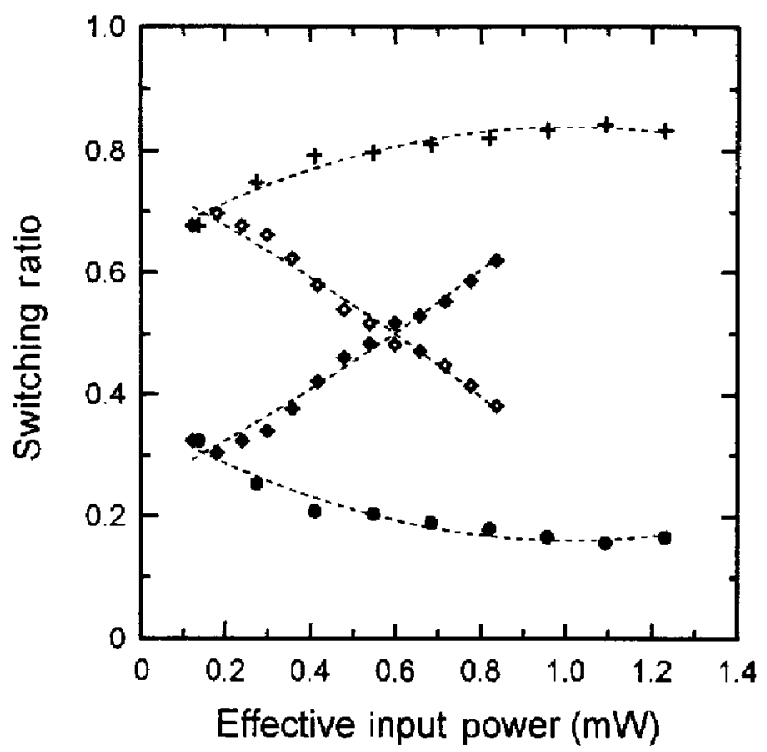

Figure 5 Switching ratios of the $(+, \bullet) \mathrm{CW}$ and $(0,+)$ picosecond signals in the $(+, 0) \mathrm{TM}$ and $(\bullet, \bullet) \mathrm{TE}$ switching modes. The input conditions are the same as those in Fig. 4.

mode; when the input power level is high, the major part of the output power appears in the TE polarization mode. A clear polarization switching can be observed. Figure 5 shows the switching ratio (i.e. the power percentage distribution between the two polarization modes) for the data shown in Fig. 4, together with the corresponding $\mathrm{CW}$ operation. We can see (Fig. 5) that the CW signal does not show any switching trend in the input power range; however, the picosecond shows clear power-dependent self-switching from $70 / 30$ to $38 / 62$. The switching power, which is defined as the input power required to make the switching ratio equal to 0.5 , is $0.6 \mathrm{~mW}$. This average power level corresponds to $7.9 \mathrm{pJ}$ pulse energy or $13.2 \mathrm{~mW} \mathrm{~cm}^{-2}$ peak intensity (the effective cross-sectional area of the waveguide was estimated to be of the order of $20 \mu \mathrm{m}^{2}$ ). Since the effective input power is higher than the real input power, as described above, the real switching pulse energy and intensity must be smaller than those values given. It is believed that by using an amplifier with a single transverse mode and a multiple quantum well structure, and by optimizing the operation conditions, the switching energy can be significantly reduced. From the data in Fig. 4, the throughput was estimated to be $14.7 \%$ at the highest input power level. Again, the real throughput must be higher than this value and can be improved by optimizing the operation conditions. In Fig. 5, a more efficient non-linear effect due to the high peak power of the picosecond signal can be easily recognized. Note that in both Figs 4 and 5, the dotted curves represent the second-order polynomial fit.

\section{Theoretical model and results}

To verify the experimental results, a theoretical model was adopted [9]. With the assumption that the TE and TM modes are in the $x$ - and $y$-directions, respectively, the electric field at the output end of the amplifier is given by:

$$
\boldsymbol{E}=A_{x} \exp \left(\mathrm{j} k_{\mathbf{r}} z\right) \hat{x}+A_{y} \exp \left(\mathrm{j} k_{y} z\right) \hat{y}
$$


Here, $A_{x}$ and $A_{y}$ are the complex amplitudes and $k_{x}$ and $k_{y}$ are the linear propagation constants in the two polarization modes, respectively. The complex amplitudes satisfy:

$$
\left(\frac{\partial}{\partial z}+\frac{1}{v_{x(y)}} \frac{\partial}{\partial t}\right) A_{x(y)}=\frac{1}{2}\left(1-\mathrm{j} \alpha_{x(y)}\right) g_{x(y)}(N) A_{x(y)}
$$

In Equation 2, $t$ is time, $v_{x}$ and $v_{y}$ are the group velocities, and $g_{x}$ and $g_{y}$ are the gain constants in the two polarization axes, respectively. Also, $\alpha_{x}$ and $\alpha_{y}$ represent the linewidth enhancement factors in the two polarizations. Because of the linear and non-linear anisotropy in gain, it is expected that $\alpha_{x}$ may be different from $\alpha_{y}$. Under the linear approximation, the gain constants are related to the carrier density, $N$, through:

$$
g_{x(y)}(N)=\Gamma_{x\{y)} a_{x(y)}\left(N-N_{x_{0}\left(y_{0} j\right.}\right)
$$

Here, $\Gamma_{x}$ and $\Gamma_{y}$ are the confinement factors, $N_{x_{0}}$ and $N_{y_{0}}$ are the transparency carrier densities, and $a_{x}$ and $a_{y}$ are the gain factors of the two polarization axes, respectively. It is the difference between $a_{x}$ and $a_{y}$ that leads to the anisotropy of gain and gain saturation. The carrier rate equation is given by:

$$
\frac{\partial N}{\partial t}=\frac{I}{q V}-\frac{N}{\tau_{\mathrm{c}}}-\frac{g_{x}(N)}{\hbar \omega_{0}}\left|A_{x}\right|^{2}-\frac{g_{y}(N)}{\hbar \omega_{0}}\left|A_{y}\right|^{2}
$$

Here, $I$ is the injection current, $q$ is the electron charge, $V$ is the effective volume, $\hbar$ is Planck's constant, $\omega_{0}$ is the signal angular frequency, and $\tau_{\mathrm{c}}$ is the carrier lifetime; the last includes the spontaneous recombinations in both polarization modes.

Combining Equations 3 and 4 leads to the following equation for the gain constants:

$$
\frac{\partial g_{x(y)}}{\partial t}=-\frac{g_{x(y)}-g_{x_{0}\left(y_{0}\right)}}{\tau_{c}}-\frac{g_{x(y)}}{E_{s x(s y)}}\left|A_{x(y)}\right|^{2}-\frac{g_{x(y)}}{E_{s x y(s y x)}}\left|A_{y(x)}\right|^{2}
$$

Here:

$$
g_{x_{0}\left(y_{0}\right)}=\Gamma_{x(y)} a_{x(y)} N_{x_{0}\left(y_{0}\right)}\left(\frac{I}{I_{x_{0}\left(y_{0}\right)}}-1\right)
$$

are the small-signal gain constants and:

$$
I_{x_{0}\left(y_{0}\right)}=q V N_{x_{0}\left(y_{0}\right)} / \tau_{\mathrm{c}}
$$

are the transparency currents. Also, $E_{s x}, E_{s y}, E_{s x y}$ and $E_{s y x}$ are the saturation energies defined by:

$$
\begin{gathered}
E_{s x(s y)}=\hbar \omega_{0} \sigma_{x(y)} / a_{x(y)} \\
E_{s x y(s y x)}=\hbar \omega_{0} \sigma_{y(x)} / a_{x(y)}
\end{gathered}
$$

where $\sigma_{x(y)}=w d / \Gamma_{x(y)}$ is the effective cross-sectional area and $w d$ is the physical crosssectional area of the amplifier.

With the following normalization procedure:

$$
\begin{aligned}
\zeta & =z / L_{\mathrm{c}} \\
\eta & =t / \tau_{\mathrm{c}} \\
q_{x(y)} & =A_{x(y)} /\left(E_{\mathbf{s} x} / \tau_{\mathrm{c}}\right)^{1 / 2}
\end{aligned}
$$


and

$$
\gamma_{x(y)}=g_{x(y)} L_{c}
$$

where $L_{\mathfrak{c}}$ is one-half the beat length of the linear birefringence. The use of Equations 10-13 makes Equation 2 become:

$$
\left(\frac{\partial}{\partial \zeta}+\delta_{x(y)} \frac{\partial}{\partial \eta}\right) q_{x(y)}=\frac{1}{2}\left(1-j \alpha_{x(y)}\right) \gamma_{x(y)} q_{x(y)}
$$

and Equation 5 becomes:

$$
\left.\frac{\partial \gamma_{x(y)}}{\partial \eta}=-\left(\gamma_{x(y)}-\gamma_{x_{0}\left(y_{0}\right)}\right)-\gamma_{x(y)} \xi_{x(y)} \mid q_{x(y)}\right)^{2}-\gamma_{x(y)} \xi_{x y(x)}\left|q_{y(x)}\right|^{2}
$$

Here $\gamma_{x_{0}\left(y_{0}\right)}=g_{x_{0}\left(y_{0}\right)} L_{\mathfrak{c}}, \delta_{x(y)}=L_{\mathfrak{c}} /\left(v_{x(y)} \tau_{c}\right), \xi_{x}=1, \xi_{y}=E_{s x} / E_{s y}+\xi_{x y}=E_{s x} / E_{s x y}$ and $\xi_{y x}=$ $E_{s x} / E_{s y x}$. Note that the anisotropy of gain saturation can be clearly seen when $\xi_{y}, \xi_{x y}$ or $\xi_{y x}$ is different from unity. Equations 14 and 15 are to be solved with appropriate parameter values.

In the experiments, several parameters concerning the optical amplifier, such as the beat length of the linear birefringence, gain constants, linewidth enhancement factors, carrier lifetime and the confinement factors, cannot be easily obtained. Therefore, simulation of the experimental situation and determination of the theoretical fit of the data are almost impossible. What we intend to do in the following is to use reasonable parameter values and show that the trends of experimental data are consistent with theoretical values.

Without loss of major physical pictures, we assume $\Gamma_{x}=\Gamma_{y}$ and hence $\sigma_{x}=\sigma_{y}$. Then, we have $\xi_{x}=\xi_{x y}=1$ and $\xi_{y}=\xi_{y x}=\frac{1}{2}$. The choice of the numerical value, $\delta_{x}=\delta_{y}=0.01$, is made under the assumption that the beat length $\left(2 L_{\mathrm{c}}\right)$ is of the order of $1 \mathrm{~mm}\left(\tau_{\mathrm{c}} \sim 10^{-9} \mathrm{~s}\right.$ and $\left.v_{x(y)} \sim 10^{8} \mathrm{~m} \mathrm{~s}^{-1}\right)$. Also, we assume $\alpha_{x}=\alpha_{y}=3$. Figure 6 shows the theoretical results of the normalized output intensity versus the normalized input intensity corresponding to the

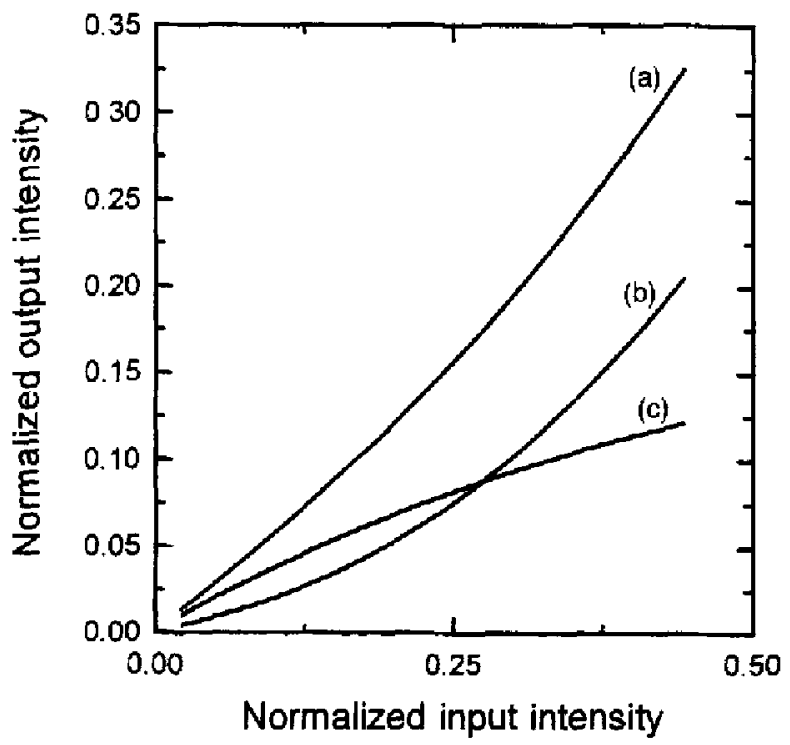

Figure 6 Theoretical results of the normalized output intensity versus the normalized input intensity corresponding to the case of Fig. 4: (a) total intensity, (b) TE mode, and (c) TM mode. 
situation in Fig. 4. The results were obtained under the assumption that the input pulse width was equal to 0.01 times the carrier lifetime and the normalized small-signal gain constants of the TE and TM modes were -1.5 and 1.0 , respectively. The negative gain constant indicates that the amplifier operates in the absorption region for the TE polarization mode. We can see that the switching trend in Fig. 6 is the same as that in Fig. 4. Figure 7 shows the corresponding switching ratios for comparison with Fig. 5. The trends in the low power range can be attributed to uncertainties in output power detection in this range. These uncertainties may be caused by several possibilities, including electroluminescence generated at wavelengths other than that of the signal and inaccuracies in reading the silicon detectors.

\section{Passive mode-locking of a semiconductor laser}

As shown in Fig. 3, input signals with different peak powers lead to different output polarization ellipses due to the anisotropy of absorption saturation. We expect a similar phenomenon with gain saturation, as proved by the self-switching results in Figs 4 and 5. If we place a polarizer orientated in the tilt angle direction of the high-peak-power signal after the amplifier, the input pulsed signal is expected to become narrower after the polarizer, because the low-power parts of the pulse are stopped by the polarizer. A similar phenomenon of pulse narrowing in an optical fibre has been used for passively mode-locking Er-doped fibre lasers [ 7,8$]$. In this section, we numerically show the possibility of passively mode-locking a semiconductor laser based on non-linear polarization rotation.

We consider a ring cavity, shown in Fig. 8, in which a travelling-wave optical amplifier with a quantum well structure is followed by a polarizer. An isolator is inserted to guarantee oneway oscillation. Equations 14 and 15 are numerically solved for each loop circulation, with careful arrangement for the time window of computations to account for the finite ring cavity length. The loop circulation starts with a long pulse that can be generated from disturbances

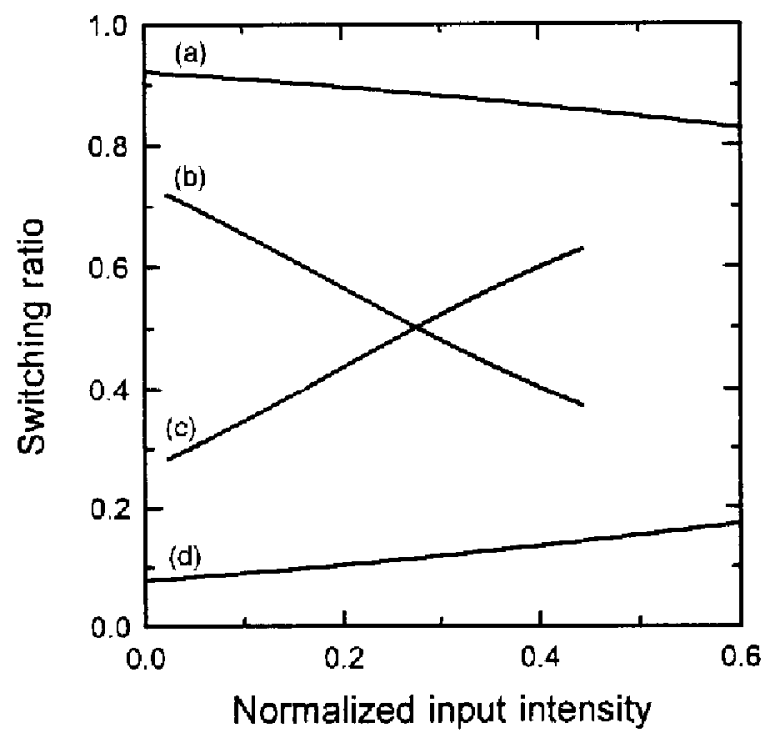

Figure 7 Theoretical results of switching ratios corresponding to the case of Fig. 5: (a) CW TM, (b) picosecond TM, (c) picosecond TE, and (d) CW TE. 


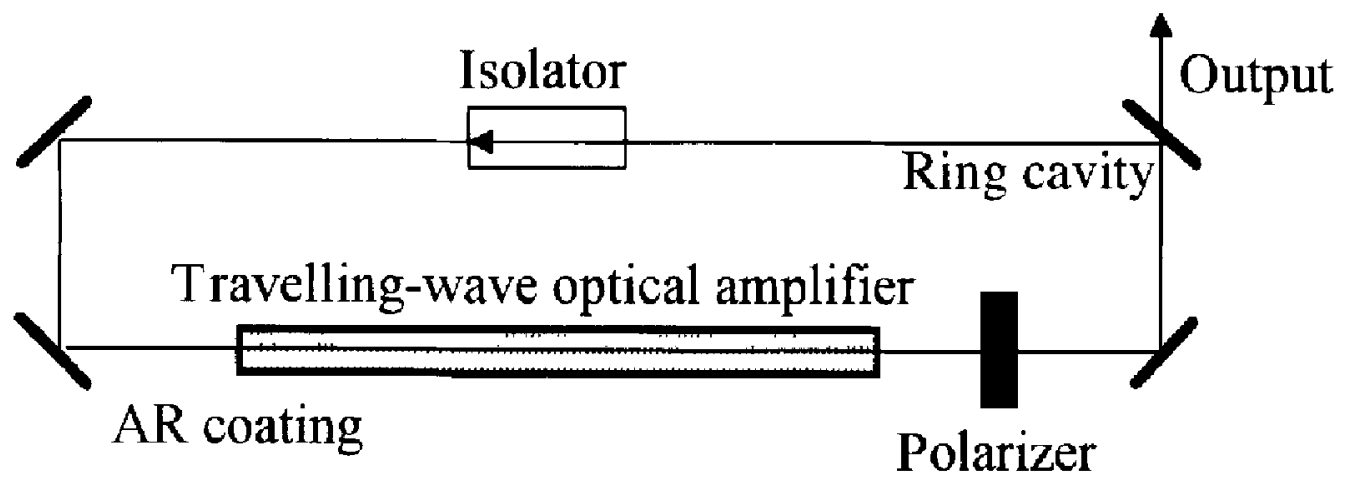

Figure 8 Ring cavity for passive mode-locking based on non-linear polarization rotation in a travelling-wave optical amplifier.

or a starting mechanism. If a systematic pulse narrowing process can be observed, passive mode-locking is possible in such a laser system. Figure 9 shows the pulse evolution with circulation in the cavity when the cavity cycle time is twice that of the carrier lifetime and the initial pulse width is 0.1 times the carrier lifetime. The amplifier is one beat length long. the normalized initial peak intensity is 10 , the output coupler has a reflectivity of 0.5 , and the normalized linear gain constant is 1.1 ( I.4) for the TE (TM) polarization mode. The values of the remaining parameters are the same as those in Section 3. In Fig. 9, we can sce that the pulse is rapidly shortened and the temporal position of the pulse is stabilized in a few tens of loops. Figure 10 demonstrates the evolution of the pulse peak position and full-width

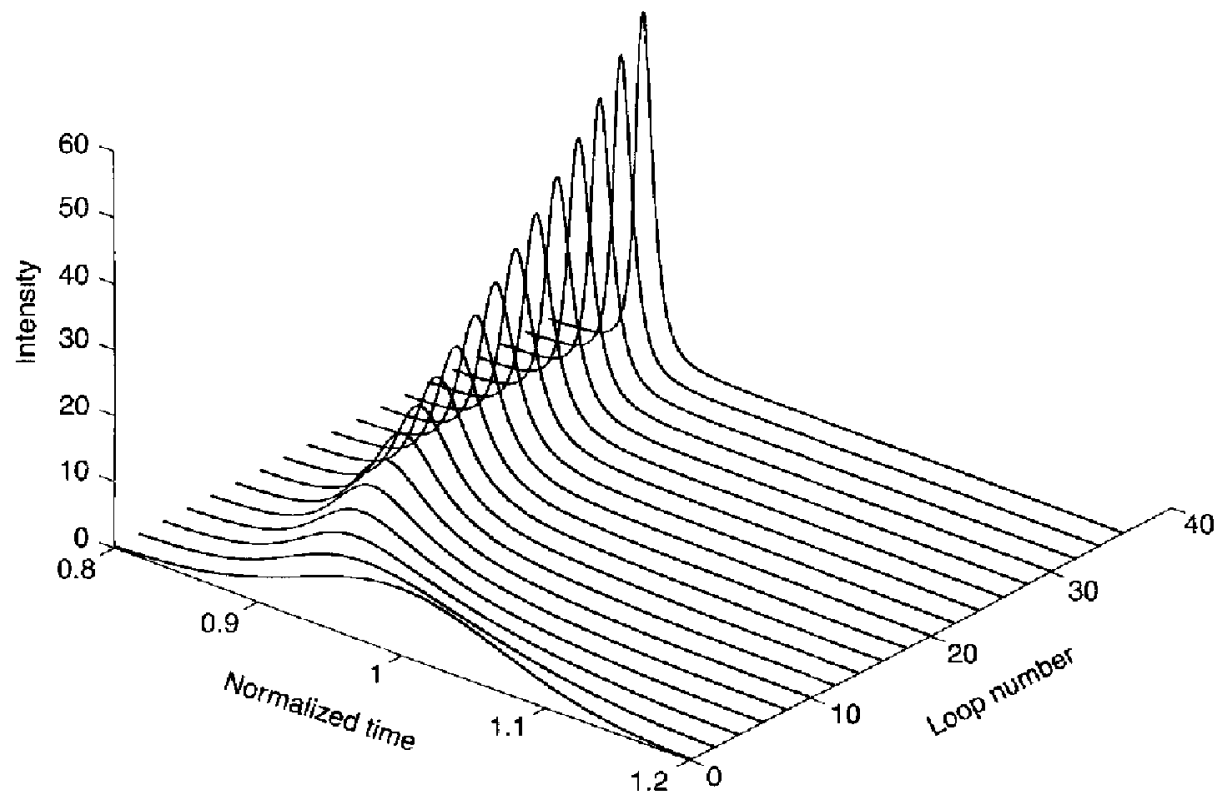

Figure 9 Pulse evolution in the ring cavity with loop circulation: a significant pulse narrowing process can be observed. 


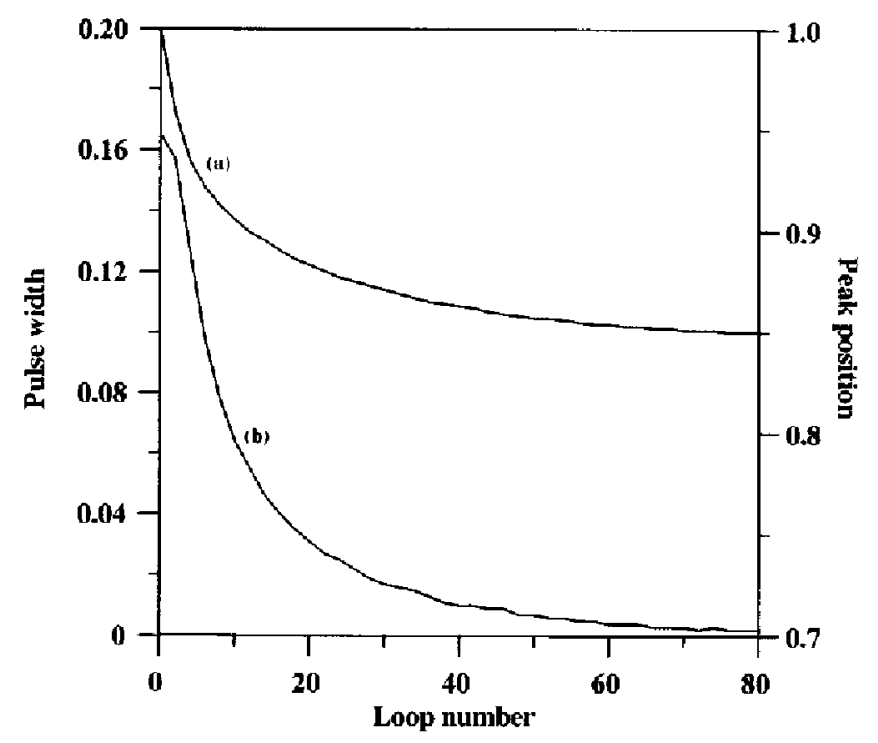

Figure 10 Evolution of the temporal position of (a) the pulse peak and (b) the FWHM pulse width.

at half-maximum (FWHM) pulse width. Also, Fig. 11 shows the evolution of the normalized pulse energy. We can see from Figs 10 and 11 that although the pulse peak position and pulse energy are stabilized after 80 circulation loops, the pulse width keeps narrowing. It is believed that the narrowing process will be stopped by some mechanisms not considered in the theoretical model, such as group-velocity dispersion and ultrafast phenomena in semiconductors. Since there is only one pulse appearing at a constant period in the cavity, what is observed in Fig. 9 implies the possibility of passive mode-locking. Detailed numerical simulations

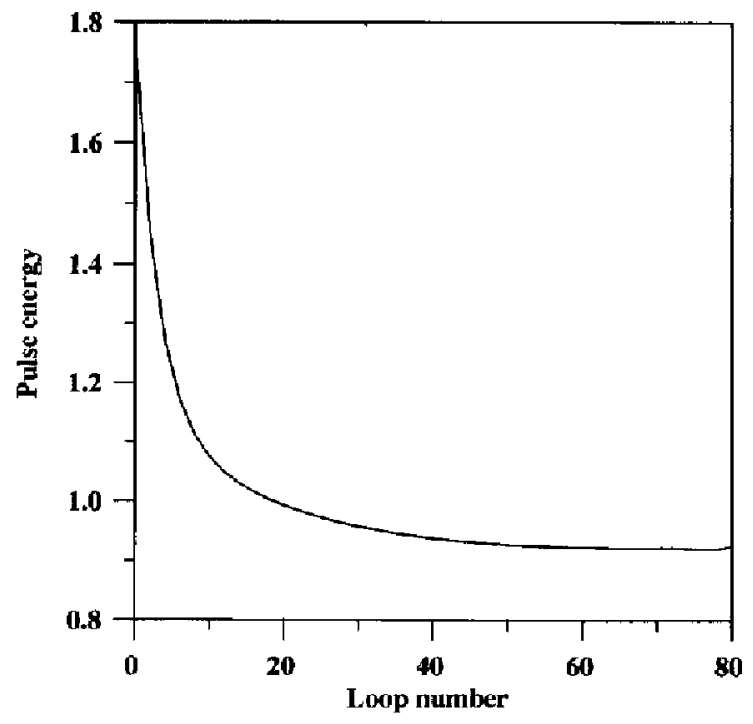

Figure 11 Evolution of the nomalized pulse energy. 
show that quite a large space exists for the variation of gain constants in the two polarization modes in which mode-locking can occur. The numerical solutions imply that implementation of such a mode-locked laser could be feasible.

\section{Conclusions}

In this paper, we have utilized the anisotropy of gain-absorption saturation in a single quantum well amplifier to show efficient non-linear polarization switching experimentally. Although the operation conditions were not optimized, the results manifested great potential of non-linear anisotropy in a semiconductor quantum well amplifier for all-optical processes. Also, we have numerically shown the feasibility of passively mode-locking semiconductor lasers based on the phenomenon of non-linear polarization rotation. With careful design of the quantum well structure and ring cawity, implementation of such a mode-locked laser should be possible.

\section{Acknowledgements}

This research was sponsored by grants NSC 84-2215-E-002-011, NSC 84-2732-E-002-002 and NSC 84-2215-E-002-012 from the National Science Council, The Republic of China.

\section{References}

1. R JIN, G. KHITROVA, H. M. GIBBS, C LOWRY and N. PEYGHAMBARIAN. Appl. Phys. Lett. 59 (1991) 3216.

2. P. LIKAMWA, A. MILLER. J. S. ROBERTS and P. N. ROBSON, Appl. Phys. Lett. 58 (1991) 2055.

3. A. VILLENEUVE, C C. YANG, P. G. WIGLEY, G. I. STEGEMAN, J S AITCHSON and C, N. IRONSIDE, Appl. Phys. Lett. 61 (1992) 147.

4. S. R FRIBERG, A. M. WEINER, Y. SLLBERBERG, B G. SFEZ and P. S. SMITH, Opt. Lett. 13 (1988) 904.

5. C. G KRALTSCHIK, G. I. STEGEMAN and R H. STOLEN, Appl. Phys, Lett, 61 (1992) 1751.

6. C C. YANG, A. VILLENEUVE, G. I. STEGEMAN, C H. LIN, H. H. LIN and I P. CHIOU, Opt. Lent. 18 (1993) 1487.

7. V. J. MATSAS, W. H. LOH and D. J RICHARDSON, IEEE Photon Technol. Lett. 5 (1993) 492.

8. H. A. HAUS, E. P. IPPEN and K TAMURA, IEEE J. Quantum Electron. 30 (1994) 200.

9. C. C. YANG, IEEE J. Quantum Electron. 29 (1993) 1091. 